\title{
Factors Associated with Short-Term Mortality After Surgical Oncologic Emergencies
}

\author{
Marianne R. F. Bosscher, MD, $\mathrm{PhD}^{1}$, Esther Bastiaannet, $\mathrm{PhD}^{2,3}$, Barbara L. van Leeuwen, $\mathrm{MD}$, $\mathrm{PhD}^{1}$, and \\ Harald J. Hoekstra, MD, PhD ${ }^{1}$ \\ ${ }^{1}$ Department of Surgical Oncology, University Medical Center Groningen, University of Groningen, Groningen, \\ The Netherlands; ${ }^{2}$ Department of Surgery, Leiden University Medical Center, Leiden University, Leiden, \\ The Netherlands; ${ }^{3}$ Department of Gerontology and Geriatrics, Leiden University Medical Center, Leiden University, \\ Leiden, The Netherlands
}

\begin{abstract}
Background. The clinical outcome of patients with oncologic emergencies is often poor and mortality is high. It is important to determine which patients may benefit from invasive treatment, and for whom conservative treatment and/or palliative care would be appropriate. In this study, prognostic factors for clinical outcome are identified in order to facilitate the decision-making process for patients with surgical oncologic emergencies.

Methods. This was a prospective registration study for patients over 18 years of age, who were consulted for surgical oncologic emergencies between November 2013 and April 2014. Multiple variables were registered upon emergency consultation, and the follow-up period was 90 days. Multivariate logistic regression analysis was performed to identify factors associated with 30- and 90-day mortality.
\end{abstract}

Results. During the study period, 207 patients experienced surgical oncologic emergencies-101 (48.8\%) men and $106(51.2 \%)$ women, with a median age of 64 years (range 19-92). The 30-day mortality was $12.6 \%$ and 90-day mortality was $21.7 \%$. Factors significantly associated with 30-day mortality were palliative intent of cancer treatment prior to emergency consultation $(p=0.006)$, Eastern Cooperative Oncology Group performance score (ECOGPS) $>0$ ( $p$ for trend: $p=0.03$ ), and raised lactate dehydrogenase $(\mathrm{LDH}) \quad(p<0.001)$. Additional factors

(C) The Author(s) 2015. This article is published with open access at Springerlink.com

First Received: 10 March 2015;

Published Online: 9 November 2015

H. J. Hoekstra, MD, PhD

e-mail: h.j.hoekstra@umcg.nl associated with 90-day mortality were low handgrip strength (HGS) $(p=0.01)$ and low albumin $(p=0.002)$. Conclusions. Defining the intent of prior cancer treatment and the ECOG-PS are of prognostic value when deciding on treatment for patients with surgical oncologic emergencies. Additional measurements of HGS, LDH, and albumin levels can serve as objective parameters to support the clinical assessment of individual prognosis.

An oncologic emergency is an acute condition experienced by a cancer patient that develops directly or indirectly from cancer or cancer treatment. ${ }^{1}$ Surgical procedures may be necessary as a (temporary) remedy. ${ }^{2}$ The clinical outcome of patients with surgical oncologic emergencies is often poor and the short-term mortality is high. $^{3-7}$ Surgical treatment can have severe complications and diminish end-of-life quality. It is important to determine which patients may benefit from invasive treatment, and for whom noninvasive treatment or referral to end-oflife care would be appropriate. Unfortunately, patient details are often limited in acute situations. ${ }^{8}$ The heterogeneity of cancer patients and surgical emergencies, as well as the wide range of treatment options, cause difficulties in decision making. Physicians often overestimate the remaining length of life of advanced cancer patients. ${ }^{9-11}$

Many studies have tried to identify prognostic factors and create prediction models for survival to assist decision making regarding cancer patients with advanced disease. ${ }^{10-16}$ Only a few studies have focused on emergency situations specifically, and even fewer studies have focused on surgical decisions in emergency situations. ${ }^{17-24}$ The aim of this study was to establish prognostic factors for the 
clinical judgment of outcome for patients with surgical oncologic emergencies in order to facilitate the decisional process regarding treatment in the acute setting. In this way, (emergency) physicians would be able to identify patients with short life expectancy, with a minimal amount of information. For this reason, parameters that do not require complex diagnostic procedures were selected for investigation. Thirty-day and ninety-day mortality were chosen as the primary and secondary endpoints.

\section{METHODS}

A prospective registration and follow-up was performed for adult cancer patients (age $>18$ years) in the University Medical Center Groningen who required surgical consultation for oncologic emergencies between 1 November 2013 and 30 April 2014. The protocol was consistent with the Declaration of Helsinki, and approval for the study was retrieved from the institutional Medical Ethics Committee.

Criteria for inclusion were consultation for surgical oncologic emergencies, which were defined as symptoms related to malignant disease or (previous) cancer treatment for which nonelective surgical consultation and/or admission was required. Patients who were consulted in the emergency room (ER), nonelectively admitted through the (surgical) outpatient clinic, transferred from other hospitals, and who required in-hospital surgical consultation when admitted for nonsurgical specialties were analyzed to meet the inclusion criteria.

Pre-existent patient characteristics were documented as parameters for disease and functional status: sex, age, oncological history, previous cancer treatment, disease status before the emergency consultation, intention of last cancer treatment, body mass index (BMI), the American Society of Anesthesiologists (ASA) classification, and the Eastern Cooperative Oncology Group performance score (ECOG-PS). The ECOG-PS has a stronger association with survival when compared with the Karnofsky Performance Status, and provides better differentiation between ambulatory and bed-ridden patients. ${ }^{25}$ The intent of cancer treatment was regarded as palliative when the patient was documented to have incurable malignant disease.

Following admission (within a maximum of $72 \mathrm{~h}$ ), parameters as proxies of illness were documented: serum leukocyte count, C-reactive protein (CRP), hemoglobin level, thrombocytes, albumin and lactate dehydrogenase (LDH). As a parameter of muscle strength, the average of three consecutive handgrip strength (HGS) measurements of the right and left hands was documented using the Jamar ${ }^{\circledR}$ Plus + dynamometer (Sammons Preston, Bolingbrook, IL, USA), which has been found to be more accurate for HGS in advanced cancer patients compared with other dynamometers. ${ }^{26}$ The deviation compared with the normative value for HGS according to age and sex was calculated for each patient, and a combination of both hands was subsequently divided into three categories: low (less than or equal to $-4.2 \mathrm{~kg}$ deviation), intermediate (between -4.1 and $1.2 \mathrm{~kg}$ deviation), and high $(\geq 1.2 \mathrm{~kg}$ deviation). When patients had undergone surgical treatment or other types of interventions before the tests could have been performed, the results were not included in the analysis since these could have been influenced by the treatment (in a positive or negative manner).

The final diagnoses of all patients were classified into different categories: obstruction, infection, clinical deterioration, gastrointestinal perforation, bleeding/thrombosis, pathological fractures, and other. ${ }^{2}$ Wound infections were scored according to the Southamptom Wound Assessment Scale. ${ }^{27}$ Intestinal obstruction with clinical evidence of tumor presence was regarded as malignant obstruction, and all other cases of (transient) intestinal obstruction in the absence of signs of disease activity were regarded as benign.

The follow-up period was 90 days after inclusion. At final follow-up, the patients' charts were analyzed for 30and 90-day mortality, and all data were processed using IBM SPSS Statistics 22 for statistical analysis (IBM Corporation, Armonk, NY, USA). The four categories of diagnoses with the highest 90-day mortality were selected; one-way analysis of variance (ANOVA) tests, a KaplanMeier plot, and log-rank tests were performed to compare means of the different parameters and survival within these four different categories. Multivariate logistic regression analysis was performed to identify factors associated with 30- and 90-day mortality for all patients.

\section{RESULTS}

During the study period, a total of 207 patients were included for analysis-101 (48.8\%) males and 106 $(51.2 \%)$ females, with a median age of 64 years (range 19-92). The most prominent type of cancer was colorectal carcinoma $(26.1 \%)$. Table 1 provides an overview of the baseline characteristics of the 207 patients. Obstruction was the most frequent surgical oncologic emergency (41.6\%), followed by infections (32.4\%) (Table 2). Of all patients, $40.1 \%$ were surgically treated within 30 days after emergency evaluation; the remaining $59.9 \%$ of patients received conservative, nonsurgical treatment.

The 30-day mortality for all patients was $12.6 \%$, and was highest for patients who presented with clinical deterioration $(42.1 \%)$, followed by patients who presented with gastrointestinal leak (25.0 \%) (Table 2). The 90-day mortality for all patients was $21.7 \%$, and was highest for 
TABLE 1 Baseline characteristics of cancer patients who required consultation for surgical oncologic emergencies $(N=207)$

\begin{tabular}{ll}
\hline Characteristic & $N \quad \%$ \\
\hline
\end{tabular}

Patient characteristics

Age (years)

$\begin{array}{lll}\leq 50 & 42 & 20.3 \\ 50-64 & 68 & 32.8 \\ 65-74 & 67 & 32.4 \\ 75+ & 30 & 14.5\end{array}$

Sex

Male

Female

$101 \quad 48.8$

ECOG performance score

0

1

2

3

4

ASA classification

1

2

3

Handgrip strength ${ }^{\mathrm{a}}$

Low $(\leq-4.2)$

Intermediate $(-4.1$ to 1.2$)$

High ( $\geq 1.2)$

\section{BMI}

$$
\begin{aligned}
& \leq 19.9 \\
& 20.0-24.9 \\
& 25.0-29.9 \\
& \geq 30.0
\end{aligned}
$$

Cancer characteristics

\section{Cancer type}

Colorectal carcinoma

Hepatobiliary

Breast cancer

Soft tissue sarcoma/GIST

Neuroendocrine tumor

Melanoma

Cervix carcinoma

Hematologic malignancy

Esophageal carcinoma

Nonmelanoma skin cancer

Lung carcinoma

Prostate carcinoma

Ovarian carcinoma

Gastric carcinoma

Other

Unknown

No cancer diagnosis

10651.2 $\begin{array}{ll}57 & 27.5\end{array}$

$85 \quad 41.1$

$\begin{array}{ll}47 & 22.7\end{array}$

$14 \quad 6.8$

$\begin{array}{ll}4 & 1.9\end{array}$

$22 \quad 10.6$

$\begin{array}{ll}136 & 65.7\end{array}$

$\begin{array}{ll}49 & 23.7\end{array}$

$\begin{array}{ll}31 & 14.9\end{array}$

$\begin{array}{ll}32 & 15.5\end{array}$

$\begin{array}{ll}32 & 15.5\end{array}$

$18 \quad 8.7$

$\begin{array}{ll}68 & 32.9\end{array}$

$\begin{array}{ll}50 & 24.1\end{array}$

$27 \quad 13.0$

426.1

$\begin{array}{ll}18 & 8.7\end{array}$

$\begin{array}{ll}14 & 6.8\end{array}$

$\begin{array}{ll}14 & 6.8\end{array}$

$13 \quad 6.3$

$\begin{array}{ll}11 & 5.3\end{array}$

$\begin{array}{ll}8 & 3.9\end{array}$

$\begin{array}{ll}8 & 3.9\end{array}$

$\begin{array}{ll}7 & 3.4\end{array}$

$\begin{array}{ll}6 & 2.9\end{array}$

$\begin{array}{ll}4 & 1.9\end{array}$

$\begin{array}{ll}3 & 1.4\end{array}$

31.4

21.0

$\begin{array}{ll}7 & 3.4\end{array}$

$14 \quad 6.8$

$\begin{array}{ll}21 & 10.1\end{array}$
TABLE 1 continued

\begin{tabular}{lrr}
\hline Characteristic & $N$ & $\%$ \\
\hline Time since cancer diagnosis & & \\
No cancer diagnosis before consultation & 21 & 10.1 \\
$<30$ days & 26 & 12.6 \\
30 days-6 months & 56 & 27.1 \\
6 months-1 year & 20 & 9.7 \\
1-2 years & 13 & 6.3 \\
2-5 years & 41 & 19.8 \\
$>5$ years & 30 & 14.5
\end{tabular}

Documented stage of treatment before surgical oncologic emergency consultation

$\begin{array}{lrl}\text { No cancer } & 21 & 10.1\end{array}$

$\begin{array}{lrl}\text { Active disease } & 132 \quad 63.8\end{array}$

$\begin{array}{llll}\text { Diagnostic stage } & 33 & 15.9\end{array}$

Receiving treatment with curative intent $\quad 5124.6$

$\begin{array}{llll}\text { Palliative stage } & 48 & 23.2\end{array}$

NED after being treated for cancer in the past $\quad 5426.1$

$\begin{array}{lll}<30 \text { days } & 19 & 9.2\end{array}$

30 days -6 months $\quad 10 \quad 4.8$

$\begin{array}{llll}6 \text { months }-1 \text { year } & 7 & 3.4\end{array}$

$1-2$ years $\quad 6 \quad 2.9$

$2-5$ years $\quad 6 \quad 2.9$

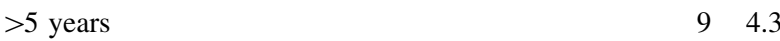

Treatment characteristics

Time since last cancer treatment

Continuously $\quad 2411.6$

$<30$ days $\quad 62 \quad 30.0$

30 days -6 months $\quad 32 \quad 15.5$

6 months-1 year $\quad 9 \quad 4.3$

$1-2$ years $\quad 15 \quad 7.2$

$2-5$ years $\quad 5 \quad 2.4$

$>5$ years $\quad 12 \quad 5.8$

No cancer treatment $\quad 48 \quad 23.2$

Intention of treatment prior to emergency consultation

$\begin{array}{lll}\text { No cancer } & 21 & 10.1 \\ \text { Diagnostic } & 32 & 15.5 \\ \text { Curative } & 49 & 23.7 \\ \text { Follow-up } & 57 & 27.5 \\ \text { Palliative } & 48 & 23.2\end{array}$

ECOG Eastern Cooperative Oncology Group, ASA American Society of Anesthesiologists, BMI body mass index, GIST gastrointestinal stromal tumor, $N E D$ no evidence of disease

${ }^{\text {a }}$ Based on the deviation of normative values according to age and $\operatorname{sex}$

patients with clinical deterioration $(52.6 \%)$, followed by patients who presented with obstruction (25.6\%). Overall, the 30-day mortality was higher for patients who underwent nonsurgical treatment $(13.7 \%)$ compared with 


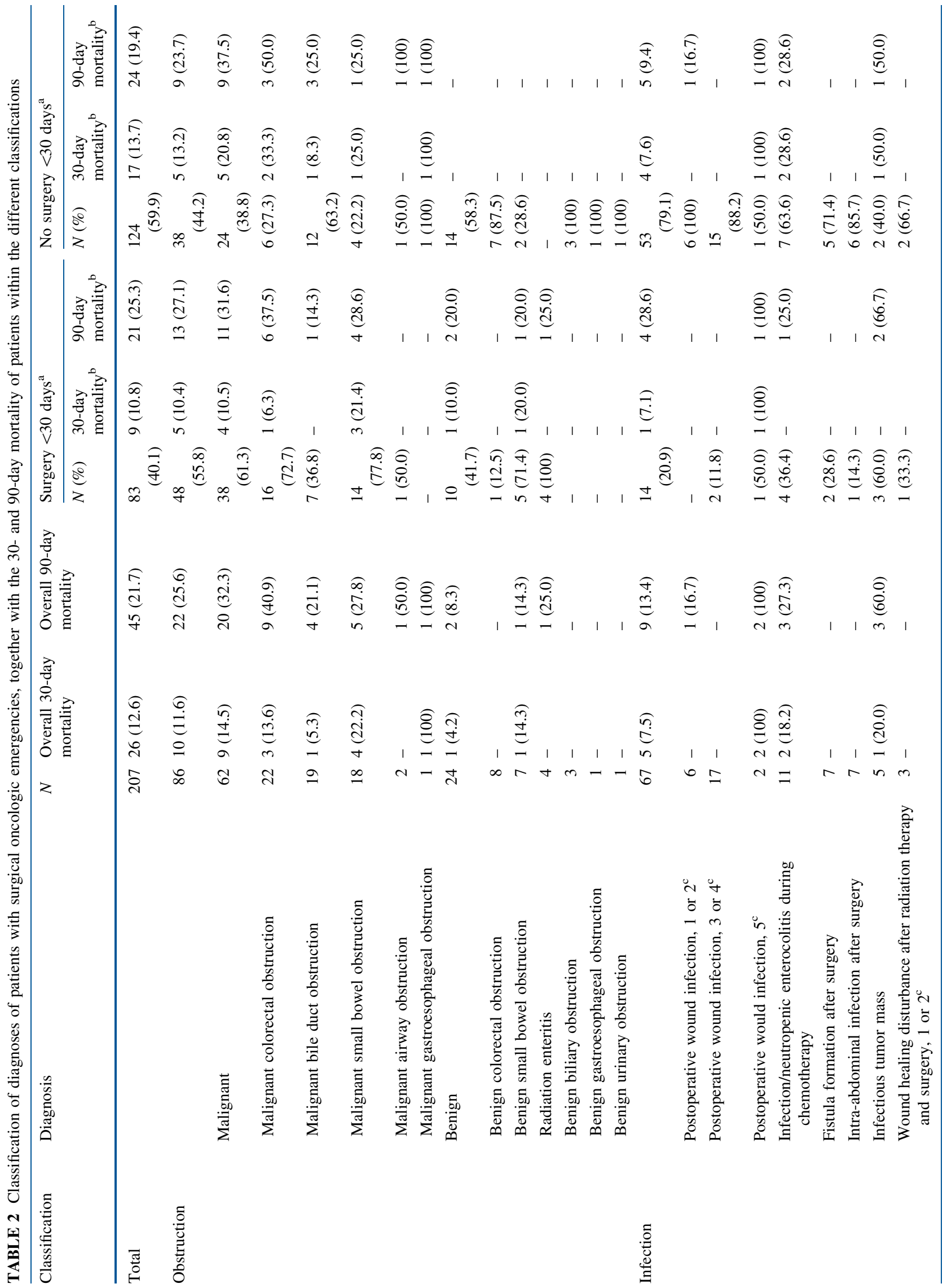




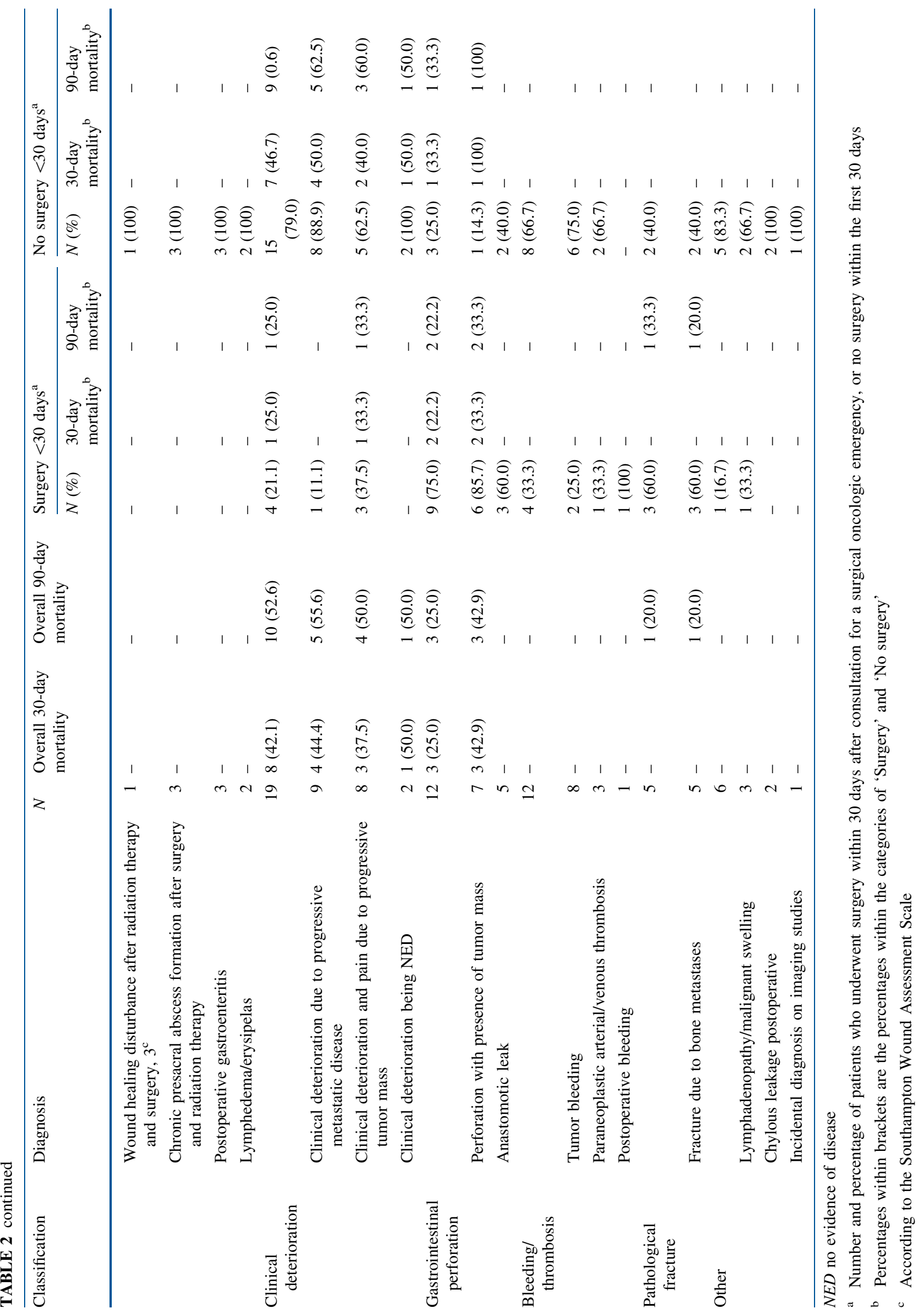


patients who underwent surgery within 30 days after consultation for surgical oncologic emergencies $(10.8 \%)$. In contrast, the overall 90-day mortality was higher for patients who underwent surgery $(25.3 \%)$ compared with patients who did not undergo surgical treatment (19.4\%).

The distribution of mortality was statistically different between the different classifications of diagnoses $(p=0.002)$. Of all patients who died after presenting with obstruction, $54.6 \%$ died between 30 and 90 days (Fig. 1).

Table 3 provides an overview of the mean values of the different parameters that were assessed upon inclusion for the four patient groups with the highest 90-day mortality. The mean ECOG-PS $(p<0.001)$, CRP $(p<0.001)$, and LDH levels $(p=0.031)$ were significantly different between the classifications of diagnoses. Other parameters showed no significant difference. The different parameters have been stratified by classification of diagnosis, and high LDH was significantly associated with 90-day mortality independent of age or sex $(p=0.01)$ of the patients who presented with obstruction $(p=0.02)$ or infection $(p=0.003)$. Other parameters showed no association for specific diagnoses.

Table 4 shows the results of the univariate and multivariate analysis of the different variables for 30- and 90day mortality. Factors significantly associated with 30-day mortality were palliative intent of treatment prior to emergency consultation $(p=0.008)$, an ECOG-PS $>0(p$ for trend: $p=0.03)$, and raised LDH $(p<0.001)$. The remaining parameters showed no association with 30-day mortality. Factors significantly associated with 90-day mortality were palliative treatment prior to emergency consultation $(p=0.01)$, ECOG-PS $>0(p=0.003)$, low HGS (less than or equal to $-4.2 \mathrm{~kg}$ deviation of the normative value; $p=0.01)$, raised LDH $(p<0.001)$, and low albumin levels $(p=0.002)$. All these factors remained significant after adjustment for age and sex.

\section{DISCUSSION}

In this study, the 30- and 90-day mortality for all patients who required consultation for surgical oncologic emergencies was 12.6 and $21.7 \%$, respectively. Factors that were significantly associated with 30-day mortality were pre-existent palliative intent of treatment, an ECOG-PS $>0$, and raised LDH. Additionally, low albumin levels and low HGS were associated with 90day mortality. These factors can all be seen as parameters of decreased functional status (i.e., performance), malnutrition, and/or advanced cancer, which are generally associated with decreased quality of life and survival. ${ }^{28-31}$
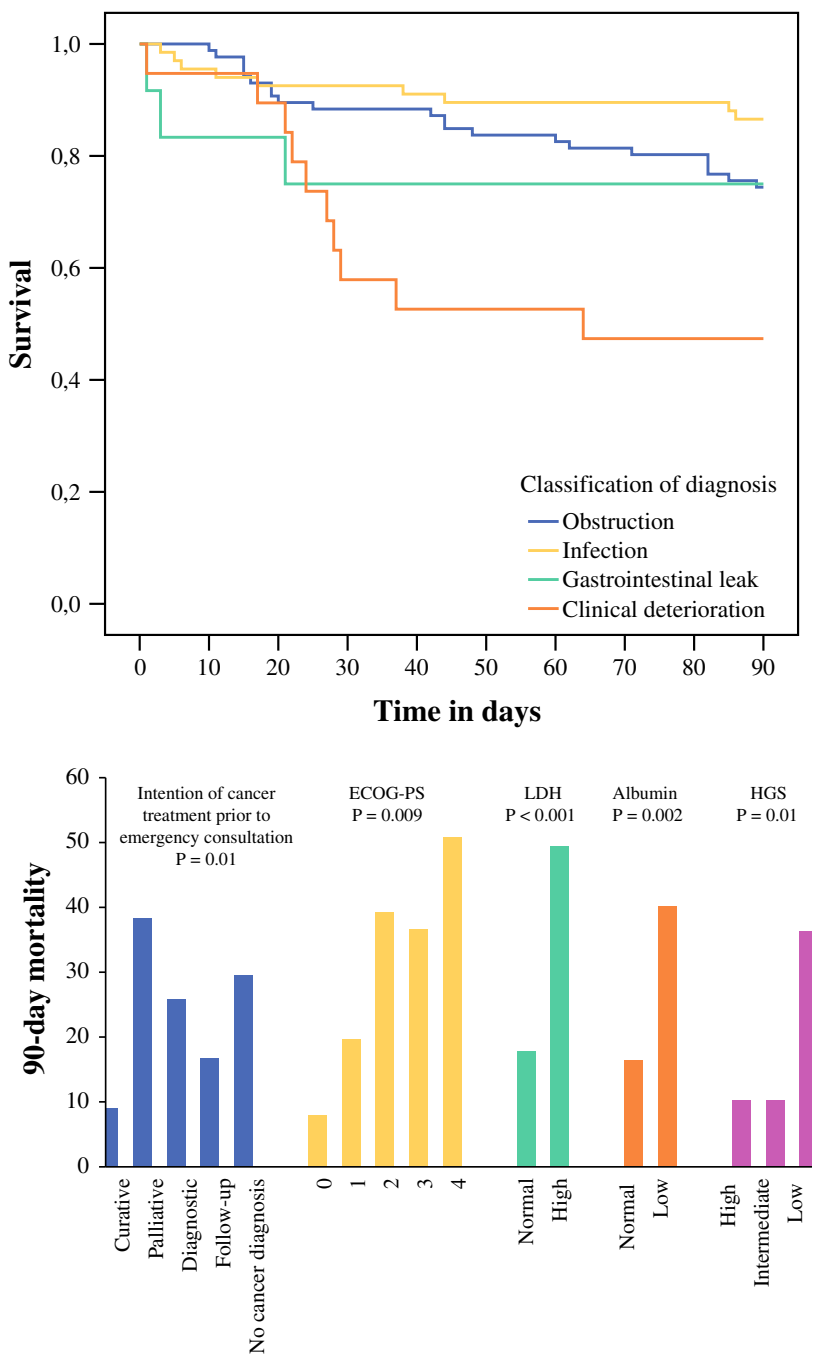

FIG. 1 Kaplan-Meier plot for survival of patients with surgical oncologic emergencies, according to the classification of diagnosis, for the classifications with the largest 90-day mortality

Advanced cancer (receiving treatment with palliative intent) and raised ECOG-PS ( $>0)$ were significantly associated with 30-day mortality. Although not specifically in an acute setting, the ECOG-PS and other functional status classification systems have already shown to be correlated with stage of disease and to be a strong predictor of survival for patients with advanced cancer. ${ }^{10,12,14,22,25,32-34}$ In one study, the ECOG-PS was the strongest predictor for mortality for patients with stage IV cancer and malignant bowel obstruction, and the median survival decreased from 222 to 63 days for patients with an ECOG-PS $>1 .^{22}$ These results underscore the importance of defining the cancer stage and functional status of a cancer patient when deciding on treatment, especially in an acute setting.

Since time is often scarce, there is a need for objective parameters that could easily be measured in order to assist in estimating the performance and predicting the clinical 
TABLE 3 Mean values of the different parameters according to the classification of diagnosis, for the classifications with the largest 90-day mortality

\begin{tabular}{|c|c|c|c|c|c|}
\hline Classification & Obstruction & Infection & Clinical deterioration & Gastrointestinal leak & $p$ value $^{\mathrm{a}}$ \\
\hline$N(\%)$ & $86(41.6)$ & $67(32.4)$ & $19(9.2)$ & $12(5.8)$ & \\
\hline ECOG-PS [Mean (range)] & $1.21(0-4)$ & $0.81(0-4)$ & $2.16(1-4)$ & $1.17(0-2)$ & 0.000 \\
\hline ASA classification [mean (range)] & $2.20(1-3)$ & $2.01(1-3)$ & $2.16(1-3)$ & $2.40(2-3)$ & 0.068 \\
\hline Distress thermometer [mean (range)] & $6.01(0.5-10)$ & $5.56(1-10)$ & $6.55(0-10)$ & $6.1(2-10)$ & 0.784 \\
\hline CRP [mean (range)] & $60(3-420)$ & $120(0-432)$ & $47(0-308)$ & $160(3-385)$ & 0.000 \\
\hline Leukocytes [mean (range)] & $11.4(3.9-38.8)$ & $11.7(0.1-61.3)$ & $11.5(5.0-19.8)$ & $13.4(7.4-21.9)$ & 0.816 \\
\hline Hemoglobin [mean (range)] & $7.6(4.7-11.4)$ & $7.1(3.2-10.2)$ & $7.7(5.7-10.0)$ & $7.4(5.3-9.6)$ & 0.152 \\
\hline Thrombocytes [mean (range)] & $349(133-961)$ & $306(14-895)$ & $370(166-559)$ & $333(146-519)$ & 0.507 \\
\hline Creatinine [mean (range)] & $86(29-412)$ & $94(35-525)$ & $81(24-182)$ & $87(36-305)$ & 0.849 \\
\hline LDH [mean (range)] & $210(0-1236)$ & $246(0-2180)$ & $405(142-1509)$ & $214(122-303)$ & 0.031 \\
\hline Albumin [mean (range)] & $36(19-49)$ & $33(16-49)$ & $36(21-45)$ & $31(17-45)$ & 0.064 \\
\hline HGS L ${ }^{\mathrm{b}}$ [mean (range)] & $-0.3(-22.6-18.6)$ & $-4.5(-18.3-3.2)$ & $-2.9(-14.7-9.5)$ & $-1.8(-3.7-0.2)$ & 0.308 \\
\hline HGS R $^{\mathrm{b}}$ [mean (range)] & $-3.4(-28.0-19.0)$ & $-6.9(-27.4-12.3)$ & $-7.7(-20.1-4.1)$ & $-6.8(-7.6--5.6)$ & 0.365 \\
\hline BMI [mean (range)] & $25.1(15.4-39.0)$ & $26.4(18.4-41.6)$ & $24.6(17.5-32.4)$ & $27.6(21.5-45.9)$ & 0.329 \\
\hline
\end{tabular}

ECOG-PS Eastern Cooperative Oncology Group performance score, ASA American Society of Anesthesiologists, $C R P$ C-reactiveprotein, $L D H$ lactate dehydrogenase, $H G S L$ hand-grip strength, left-hand, $H G S R$ hand-grip strength, right-hand, $B M I$ body mass index, $A N O V A$ analysis of variance

a One-way ANOVA

b Based on the deviation of the normative according to age and sex

outcome of surgical oncologic emergencies. When patients undergo invasive treatment such as surgery, it is essential that the patient is able to recover from this invasive procedure and that the procedure itself does not reduce the patient's quality of life.

Blood tests and other laboratory tests are often routinely performed, especially for patients who visit the ER. In this study, raised LDH was significantly associated with 30-day mortality and low albumin was associated with 90-day mortality after surgical oncologic emergencies. The prognostic value of raised LDH and low albumin for terminally ill cancer has been confirmed by other studies; however, it has not been widely investigated in an acute setting. ${ }^{22,25,30,35-37}$ The remaining blood markers that were analyzed for this study (i.e., leukocytes, hemoglobin, CRP, thrombocytes, and creatinine) were not generally associated with 30- or 90-day mortality after surgical oncologic emergencies.

In general, sepsis (often accompanied with elevated serum CRP levels) after emergency surgery is associated with postoperative mortality. ${ }^{38}$ In this study, the mortality for patients with high CRP levels was lower compared with patients from other groups. Even within the classification of infection, high CRP levels were not associated with 30or 90-day mortality, possibly due to the fact that most patients within the group of infections had postoperative wound infections, which are often conservatively treated with antibiotics or drainage. Furthermore, the number of patients with severe sepsis was relatively small. Studies on electively treated cancer patients found that CRP is associated with malignant potential and tumor stage, and thus general prognosis. ${ }^{32,39,40} \mathrm{CRP}$ has been found to increase significantly $1-2$ weeks prior to death. ${ }^{41}$ In a study evaluating CRP levels in patients with advanced cancer visiting the ER, CRP was considered to be an independent predictor for 14-day mortality, but the specific diagnoses and the effect of antimicrobial treatment were not specified. ${ }^{23}$

In the present study, low HGS (less than or equal to $-4.2 \mathrm{~kg}$ deviation of the normative value) was associated with 90-day mortality. HGS has been found be a measure of muscle function, cachexia, and malnutrition in several cancer populations, and a better predictor of clinical outcome than measuring the appendicular muscle mass. ${ }^{28,31,42-48}$ HGS has been associated with a significantly lower BMI, hemoglobin, and albumin, and increased ECOG-PS. Malnutrition and low HGS have further been associated with an increased length of hospital stay and mortality. In one study, patients with a decline of HGS to less than a 10th percentile of normative values had statistically shorter survival compared with patients with higher HGS, independent of age, sex, oncological treatment, and cancer type. ${ }^{28,43}$ Nevertheless, HGS less than a 10th percentile of the normative value is an extensive decline in strength, and the survival period was relatively long. The 


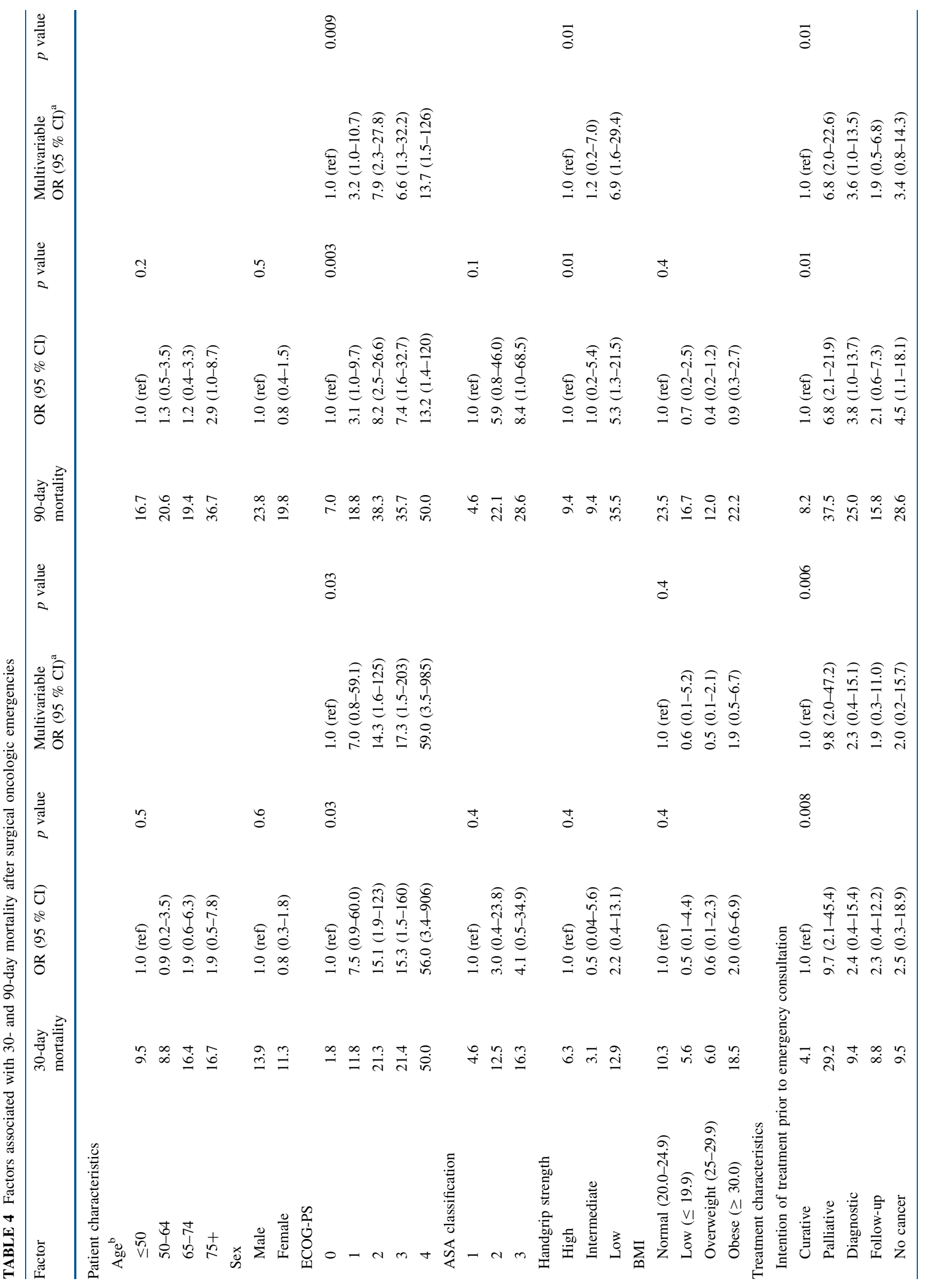




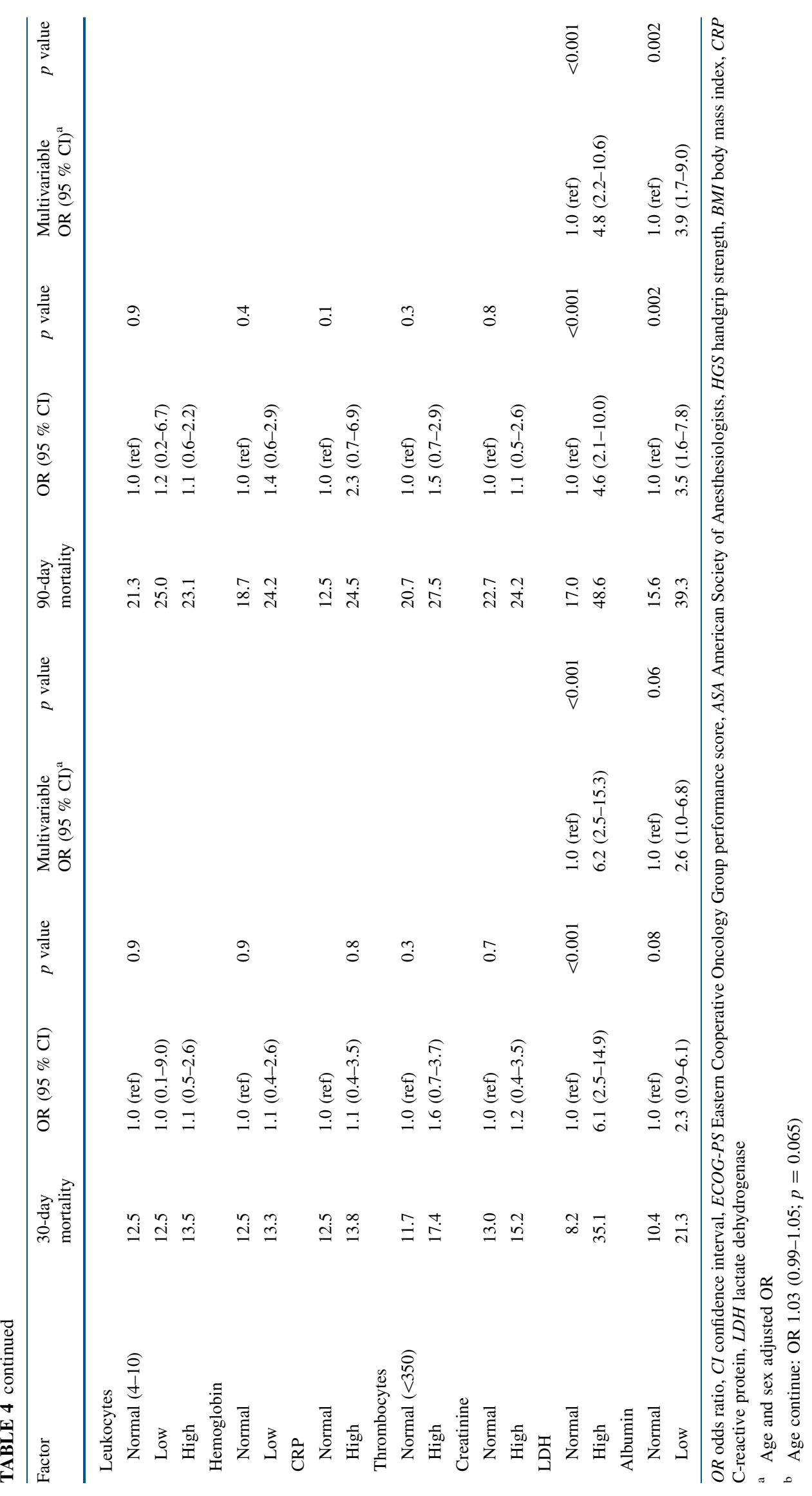


current study has found that even a smaller decline in HGS (less than or equal to $-4.2 \mathrm{~kg}$ deviation of the normative value) was already associated with 90-day mortality.

The results of both previous studies and the current study confirm that HGS can be seen as a measurement of functional status and that low HGS is associated with poor clinical outcome. To our knowledge, this is the first study evaluating the clinical value of HGS specifically for patients who are consulted for surgical oncologic emergencies. Early measurement of HGS in patients with surgical oncologic emergencies can be of value in order to identify patients with advanced cancer and poor functional status who require referral to palliative care. When dynamometers become generally available at the ER and hospital wards, HGS could be an easy measure for patients who require prompt decisions. Unfortunately, HGS could not be measured prior to treatment in every patient. Many patients were admitted and treated outside office hours when research personnel were not available, and some patients were not hospitalized after visiting the ER. For other patients, the measurements were not performed at the time of the first consultation but after 1 or 2 days of admission, and therefore the hand grip strength could have been influenced. Possibly, when more HGS measurements would have been performed over a shorter term after the first consultation, a stronger association with short-term mortality may have been found. Further studies are necessary to validate the prognostic value of HGS in the emergency setting.

Clinical functional status scoring systems and other parameters have already been incorporated in multiple prediction models for survival of terminally ill cancer patients ${ }^{10,25,36,49}$; however, none of these models have been evaluated for patients with (surgical) oncologic emergencies. The results of this study confirm that defining the ECOG-PS and cancer stage, as well as measurements of LDH, albumin, and HGS, are of prognostic value for patients with surgical oncologic emergencies with respect to 30- and 90-day mortality. When deciding on the extent of treatment, the main goal of treatment should be (temporary) solution of the emergency without reducing survival or quality of life. Being able to recognize patients who are at the end of life could prevent unnecessary investigations and expensive treatment, and preserve overall patient satisfaction. The parameters investigated in this study will not predict a specific remaining duration of life, but the combination of prognostic factors found in this study can support the clinical judgment of a physician who is confronted with surgical oncologic emergencies.

Only two other studies have investigated factors associated with mortality in patients with surgical oncologic emergencies. ${ }^{17,24}$ Dumont et al. created a preoperative normogram for decision making in surgical oncologic emergencies, which included the ECOG-PS and albumin level (both confirmed by the current study), as well as the
Portsmouth Physiological and Operative Severity Score for the enumeration of Mortality and Morbidity (P-POSSUM). ${ }^{17}$ The major drawback of the P-POSSUM is that this score is not designed for the acute setting and requires comprehensive preoperative diagnostic studies. A study by Roses et al. has identified ASA classification $>3$ and albumin as independent predictors for 30-day mortality. ${ }^{24}$ Active malignant disease, a tumor-related emergency, ASA $>3$, and raised creatinine were independent predictors of decreased overall survival. For patients in the current study, the pre-existent ASA classification was assessed and did not include any patient classified higher than 3. The ASA classification was not associated with short-term mortality. The ECOG-PS seems to be a better indicator for clinical outcome in the acute setting.

The cohort of patients in this study represents a very heterogeneous population, and patients experienced a wide range of emergencies with various severities. As this was an observational study, patients were not selected or randomized for invasive or noninvasive treatment according to the prognostic factors. The treatment instituted for each patient was dependent on the decisions of the physicians involved during admission, and was not influenced by this study. Furthermore, because of the amount of missing values and the relatively small numbers of patients in the different subgroups, we were not able to create a solid prediction model. For this reason, only factors that showed significant association with 30 - and 90-day mortality were shown. However, we believe that the combination of these parameters can assist in estimating a patient's prognosis in the acute setting.

\section{CONCLUSIONS}

Consultation for surgical oncologic emergencies can be a sign of advanced disease, and outcome is often poor. Being able to recognize patients who are at the end of life would prevent unnecessary investigations, expensive treatment, and preserve patient satisfaction. There is a need for parameters that can easily be measured in order to assist in predicting the clinical outcome. Defining the intention of prior cancer treatment and the ECOG-PS are of prognostic value when deciding on the extent of treatment for patients with surgical oncologic emergencies. Additional measurements of LDH and albumin levels, as well as HGS, can serve as objective prognostic parameters to assist the clinical judgment of individual outcome. This would aid the decision-making process in the acute setting. In this way, patient-tailored treatment can be instituted and overtreatment can be prevented. Further studies will be necessary to validate the prognostic value of these and possible additional parameters in the acute oncology setting. 
DISCLOSURES Marianne R. F. Bosscher received a research grant from the Groningen Melanoma and Sarcoma Foundation. No other funding was received for this study, and there are no commercial interests.

OPEN ACCESS This article is distributed under the terms of the Creative Commons Attribution 4.0 International License (http:// creativecommons.org/licenses/by/4.0/), which permits unrestricted use, distribution, and reproduction in any medium, provided you give appropriate credit to the original author(s) and the source, provide a link to the Creative Commons license, and indicate if changes were made.

\section{REFERENCES}

1. Cervantes A, Chirivella I. Oncological emergencies. Ann Oncol. 2004;15 Suppl 4:iv299-306.

2. Bosscher MR, van Leeuwen BL, Hoekstra HJ. Surgical emergencies in oncology. Cancer Treat Rev. 2014;40(8):1028-36.

3. Barbera L, Taylor C, Dudgeon D. Why do patients with cancer visit the emergency department near the end of life? CMAJ. 2010;182(6):563-8.

4. Barnett A, Cedar A, Siddiqui F, Herzig D, Fowlkes E, Thomas CR Jr. Colorectal cancer emergencies. J Gastrointest Cancer. 2013;44(2):132-42.

5. Porta M, Fernandez E, Belloc J, Malats N, Gallen M, Alonso J. Emergency admission for cancer: a matter of survival? $\mathrm{Br} J$ Cancer. 1998;77(3):477-84.

6. Geraci JM, Tsang W, Valdres RV, Escalante CP. Progressive disease in patients with cancer presenting to an emergency room with acute symptoms predicts short-term mortality. Support Care Cancer. 2006;14(10):1038-45.

7. Bosscher MR, van Leeuwen BL, Hoekstra HJ. Mortality in emergency surgical oncology. Ann Surg Oncol. 2015;22(5):1577-84.

8. Downing A, Aravani A, Macleod U, Oliver S, Finan PJ, Thomas JD, et al. Early mortality from colorectal cancer in England: a retrospective observational study of the factors associated with death in the first year after diagnosis. Br J Cancer. 2013;108(3):681-5.

9. Glare P, Virik K, Jones M, Hudson M, Eychmuller S, Simes J, et al. A systematic review of physicians' survival predictions in terminally ill cancer patients. BMJ. 2003;327(7408):195-8.

10. Llobera J, Esteva M, Rifa J, Benito E, Terrasa J, Rojas C, et al. Terminal cancer. Duration and prediction of survival time. Eur J Cancer. 2000;36(16):2036-43.

11. Vigano A, Donaldson N, Higginson IJ, Bruera E, Mahmud S, SuarezAlmazor M. Quality of life and survival prediction in terminal cancer patients: a multicenter study. Cancer. 2004;101(5):1090-8.

12. Liu Y, Zhang PY, Na J, Ma C, Huo WL, Han L, et al. Prevalence, intensity, and prognostic significance of common symptoms in terminally ill cancer patients. J Palliat Med. 2013;16(7):752-7.

13. Knops AM, Legemate DA, Goossens A, Bossuyt PM, Ubbink DT. Decision aids for patients facing a surgical treatment decision: a systematic review and meta-analysis. Ann Surg. 2013;257(5):860-6.

14. Stone PC, Lund S. Predicting prognosis in patients with advanced cancer. Ann Oncol. 2007;18(6):971-6.

15. Selby D, Chakraborty A, Lilien T, Stacey E, Zhang L, Myers J. Clinician accuracy when estimating survival duration: the role of the patient's performance status and time-based prognostic categories. J Pain Symptom Manag. 2011;42(4):578-88.

16. Yanneo EG. Determining prognosis and predicting survival in endof-life care. Curr Opin Support Palliat Care. 2009;3(3):203-6.

17. Dumont F, Mazouni C, Bitsakou G, Morice P, Goéré D, Honoré $\mathrm{C}$, et al. A pre-operative nomogram for decision making in oncological surgical emergencies. J Surg Oncol. 2014;109(7): 721-5.

18. Ramchandran KJ, Shega JW, Von Roenn J, Schumacher M, Szmuilowicz E, Rademaker A, et al. A predictive model to identify hospitalized cancer patients at risk for 30-day mortality based on admission criteria via the electronic medical record. Cancer. 2013;119(11):2074-80.

19. Bottle A, Tsang C, Parsons C, Majeed A, Soljak M, Aylin P. Association between patient and general practice characteristics and unplanned first-time admissions for cancer: observational study. Br J Cancer. 2012;107(8):1213-9.

20. Kwok AC, Lipsitz SR, Bader AM, Gawande AA. Are targeted preoperative risk prediction tools more powerful? A test of models for emergency colon surgery in the very elderly. $J \mathrm{Am}$ Coll Surg. 2011;213(2):220-5.

21. Lee JS, Kwon OY, Choi HS, Hong HP, Ko YG. Application of the Sequential Organ Failure Assessment (SOFA) score in patients with advanced cancer who present to the ED. Am J Emerg Med. 2012;30(2):362-6.

22. Wright FC, Chakraborty A, Helyer L, Moravan V, Selby D. Predictors of survival in patients with non-curative stage IV cancer and malignant bowel obstruction. J Surg Oncol. 2010; 101(5):425-9.

23. Lee JS, Kwon OY, Choi HS, Hong HP, Ko YG. Serum C-reactive protein level is a predictive factor for 14-day mortality of patients with advanced cancer who present to the emergency department with acute symptoms. Acad Emerg Med. 2011;18(4):440-2.

24. Roses RE, Tzeng CW, Ross MI, Fournier KF, Abbott DE, You YN. The palliative index: predicting outcomes of emergent surgery in patients with cancer. J Palliat Med. 2014;17(1):37-42.

25. Vigano A, Bruera E, Jhangri GS, Newman SC, Fields AL, Suarez-Almazor ME. Clinical survival predictors in patients with advanced cancer. Arch Intern Med. 2000;160(6):861-8.

26. Trutschnigg B, Kilgour RD, Reinglas J, Rosenthall L., Hornby L, Morais JA, et al. Precision and reliability of strength (Jamar vs. Biodex handgrip) and body composition (dual-energy X-ray absorptiometry vs. bioimpedance analysis) measurements in advanced cancer patients. Appl Physiol Nutr Metab. 2008;33(6):1232-9.

27. Bailey IS, Karran SE, Toyn K, Brough P, Ranaboldo C, Karran SJ. Community surveillance of complications after hernia surgery. BMJ. 1992;304(6825):469-71.

28. Norman K, Stobaus N, Smoliner C, Zocher D, Scheufele R, Valentini L, et al. Determinants of hand grip strength, knee extension strength and functional status in cancer patients. Clin Nutr. 2010;29(5):586-91.

29. Puccio M, Nathanson L. The cancer cachexia syndrome. Semin Oncol. 1997;24(3):277-87.

30. Wallengren O, Lundholm K, Bosaeus I. Diagnostic criteria of cancer cachexia: relation to quality of life, exercise capacity and survival in unselected palliative care patients. Support Care Cancer. 2013;21(6):1569-77.

31. Cruz-Jentoft AJ, Baeyens JP, Bauer JM, Boirie Y, Cederholm T, Landi F, et al. Sarcopenia: European consensus on definition and diagnosis: report of the European Working Group on Sarcopenia in Older People. Age Ageing. 2010;39(4):412-23.

32. Mantovani G, Maccio A, Madeddu C, Mura L, Gramignano G, Lusso MR, et al. Quantitative evaluation of oxidative stress, chronic inflammatory indices and leptin in cancer patients: correlation with stage and performance status. Int $J$ Cancer. 2002;98(1):84-91.

33. Cesari M, Cerullo F, Zamboni V, Di Palma R, Scambia G, Balducci $\mathrm{L}$, et al. Functional status and mortality in older women with gynecological cancer. J Gerontol A Biol Sci Med Sci. 2013;68(9):1129-33. 
34. Chow E, Harth T, Hruby G, Finkelstein J, Wu J, Danjoux C. How accurate are physicians' clinical predictions of survival and the available prognostic tools in estimating survival times in terminally ill cancer patients? A systematic review. Clin Oncol ( $R$ Coll Radiol). 2001;13(3):209-18.

35. Vigano A, Dorgan M, Buckingham J, Bruera E, Suarez-Almazor ME. Survival prediction in terminal cancer patients: a systematic review of the medical literature. Palliat Med. 2000;14(5):363-74.

36. Glare P, Sinclair C, Downing M, Stone P, Maltoni M, Vigano A. Predicting survival in patients with advanced disease. Eur $J$ Cancer. 2008;44(8):1146-56.

37. Pasanisi F, Orban A, Scalfi L, Alfonsi L, Santarpia L, Zurlo E, et al. Predictors of survival in terminal-cancer patients with irreversible bowel obstruction receiving home parenteral nutrition. Nutrition. 2001;17(7-8):581-4.

38. Mallol M, Sabate A, Dalmau A, Koo M. Risk factors and mortality after elective and emergent laparatomies for oncological procedures in 899 patients in the intensive care unit: a retrospective observational cohort study. Patient Saf Surg. 2013;7(1):29.

39. Nozoe T, Matsumata T, Kitamura M, Sugimachi K. Significance of preoperative elevation of serum C-reactive protein as an indicator for prognosis in colorectal cancer. Am J Surg. 1998;176(4):335-8.

40. Kersten C, Louhimo J, Algars A, Lahdesmaki A, Cvancerova M, Stenstedt $\mathrm{K}$, et al. Increased C-reactive protein implies a poorer stagespecific prognosis in colon cancer. Acta Oncol. 2013;52(8):1691-98.

41. Suh SY, Ahn HY. A prospective study on C-reactive protein as a prognostic factor for survival time of terminally ill cancer patients. Support Care Cancer. 2007;15(6):613-20.

42. Lauretani F, Russo CR, Bandinelli S, Bartali B, Cavazzini C, Di Iorio A, et al. Age-associated changes in skeletal muscles and their effect on mobility: an operational diagnosis of sarcopenia. $J$ Appl Physiol. 2003;95(5):1851-60.

43. Kilgour RD, Vigano A, Trutschnigg B, Lucar E, Borod M, Morais JA. Handgrip strength predicts survival and is associated with markers of clinical and functional outcomes in advanced cancer patients. Support Care Cancer. 2013;21(12):3261-70.

44. Di Monaco M, Castiglioni C, De Toma E, Gardin L, Giordano S, Di Monaco R, et al. Handgrip strength but not appendicular lean mass is an independent predictor of functional outcome in hipfracture women: a short-term prospective study. Arch Phys Med Rehabil. 2014;95:1719-24.

45. Burden ST, Hill J, Shaffer JL, Todd C. Nutritional status of preoperative colorectal cancer patients. J Hum Nutr Diet. 2010;23(4):402-7.

46. Sanchez-Rodriguez D, Marco E, Miralles R, Fayos M, Mojal S, Alvarado M, et al. Sarcopenia, physical rehabilitation and functional outcomes of patients in a subacute geriatric care unit. Arch Gerontol Geriatr. 2014;59(1):39-43.

47. Chung CJ, Wu C, Jones M, Kato TS, Dam TT, Givens RC, et al. Reduced handgrip strength as a marker of frailty predicts clinical outcomes in patients with heart failure undergoing ventricular assist device placement. J Card Fail. 2014;20(5):310-5.

48. Mendes J, Alves P, Amaral TF. Comparison of nutritional status assessment parameters in predicting length of hospital stay in cancer patients. Clin Nutr. 2014;33(3):466-70.

49. Maltoni M, Caraceni A, Brunelli C, Broeckaert B, Christakis N, Eychmueller S, et al. Prognostic factors in advanced cancer patients: evidence-based clinical recommendations. A study by the Steering Committee of the European Association for Palliative Care. J Clin Oncol. 2005;23(25):6240-8. 